\title{
Mecanización agrícola de la piña en el valle de Satipo, Fundo Santa Teresa
}

\author{
Agricultural mechanization of pineapple in the valley of Satipo, \\ Santa Teresa Farm
}

\section{Francisco J. Rojas Alejandro ${ }^{1}$}

\section{Resumen}

El presente trabajo, contiene información recopilada del cultivo de piña Golden en el valle de Satipo, durante las múltiples visitas realizadas al fundo Santa Teresa de la Universidad Nacional Agraria La Molina, como viajes de prácticas del curso de Mecanización Agrícola. La idea fundamental es brindar información al lector interesado en desarrollar el cultivo de piña Golden, una visión de las diferentes actividades que intervienen en su producción, así como brindar conocimiento del avance obtenido por la Universidad Nacional Agraria La Molina y su metodología de cultivo en este campo. Asimismo, que pueda servir como incentivo al cambio del cultivo de la coca con uno alternativo de futuro económico promisorio.

Palabras clave: piña; mecanización; cultivo alternativo; fundo Santa Teresa.

\begin{abstract}
The present research contains compiled information about golden pineapple crops in Satipo's valley, during the multiple visits made as part to the Agricultural Mechanization practices to the Santa Teresa farm, which belongs to the University National Agraria La Molina. The fundamental idea is to offer information to the reader interested in developing the golden pineapple crop, a view of the different activities involved in its production, as well as providing information about the advances obtained by the UNALM and the methodology of farming in this field. Likewise it could function as an incentive to change from the coca crop to an alternative crop with a promising economic future.
\end{abstract}

Keywords: pineapple; mechanization; alternative crop; Santa Teresa farm.

\section{Introducción}

La Provincia de Satipo se sitúa en la parte oriental del departamento de Junín, bajo la administración del Gobierno Regional de Junín, en la selva central del Perú. Limita al norte con el departamento de Pasco, al este con los departamentos de Ucayali y Cusco, al sur con el departamento de Ayacucho y al oeste con las provincias de Chanchamayo, Jauja, Concepción y Huancayo.

El fundo Santa Teresa está ubicado en el kilómetro 110 de la carretera que une las ciudades de La Merced y Satipo, en el distrito de Río Negro de la provincia de Satipo del departamento de Junín, con una extensión aproximada de 203 hectáreas.

La agricultura, como actividad económica predominante en la provincia de Satipo, se realiza en 97 mil hectáreas, mientras que la ganadería en 31 mil hectáreas. El mayor uso de tierras agrícolas se da en condiciones de secano y muy pocas tierras se encuentran bajo riego. Sin embargo, existe un enorme potencial de tierras para el desarrollo de las actividades forestales. El $93 \%$ de suelos mayormente son tierras forestales y de protección, solo el $5 \%$ son para uso agropecuario. Sin embargo, la superficie agrícola actual es de 401.340 hectáreas, lo que indica que los suelos han sido sobre usados y por lo tanto urge la realización de proyectos de reforestación. Lamentablemente, el uso de las tierras en el ámbito de la provincia se efectúa sin tener en cuenta las recomendaciones técnicas para el uso adecuado de las mismas.

En todo el valle de la selva central, incluido Satipo, es tangible el problema del narcotráfico que, en simbiosis con el terrorismo, va ganado terreno rápidamente, sacando provecho de las necesidades insatisfechas de los pobladores, quienes al no recibir asistencia oportuna del estado ni orientación efectiva para la producción de cultivos alternativos, caen en las garras de este ilícito negocio. Este problema, aunado a la falta de protección de las autoridades, los obliga a estos a dedicarse al cultivo de la coca, cuya producción de hojas con su rentabilidad ayuda a paliar sus necesidades inmediatas.

La piña Golden, como cultivo alternativo por su producción y rendimiento económico, debido a factores climáticos favorables del valle de la selva central, está demostrando una sostenibilidad en el tiempo y posibilidad de convertirse en solución de la agricultura incipiente de este lugar. El fundo Santa Teresa de la Universidad Nacional Agraria La Molina viene cultivando ya casi una década esta fruta con resultados aceptables, adaptando algunas herramientas y

${ }^{1}$ Departamento de Mecanización Agrícola. Universidad Nacional Agraria La Molina, Lima (Perú). Email: frojas@lamolina.edu.pe 
equipos apropiados para la producción de este cultivo. Este trabajo es presentado por iniciativa del curso de Mecanización Agrícola, resultado de las múltiples visitas en diferentes tiempos y etapas de la producción de piña Golden, recopilando las diferentes actividades presentes en su producción, con la finalidad de dar una visión global al futuro cultivador de este producto, de lo que significa la producción de piña Golden y, asimismo, incentivar su producción en reemplazo de la hoja de coca. Asimismo, fomentar la curiosidad de nuestros técnicos en este cultivo a fin de recibir una mayor orientación sobre el tema.

Los objetivos de esta investigación son 1) dar a conocer el uso de los diferentes equipos y maquinaria empleados en la preparación de tierras, siembra, mantenimiento de cultivos y control fitosanitario, así como en la cosecha y otras técnicas para el cultivo de la piña, empleados en el fundo Santa Teresa; 2) brindar conocimiento de algunos productos químicos empleados en las diferentes etapas del cultivo y 3 ) incentivar la siembra de la piña Golden como cultivo alternativo en el valle de Satipo.

\section{Materiales y métodos}

La metodología empleada por el fundo Santa Teresa para el cultivo de la piña Golden es innovadora y propia de los técnicos de la Universidad Nacional Agraria La Molina.

\section{Cultivo: Piña Golden}

- Familia: Bromeliaceae

- Nombre científico: Ananas sativus (Lindl) Schult.

- Origen: zonas tropicales de Brasil.

- Planta: vivaz, con una base formada por la unión compacta de varias hojas formando una roseta. De las axilas de las hojas pueden surgir retoños con pequeñas rosetas basales, que facilitan la reproducción vegetativa de la planta.

- Tallo: después de uno o dos años crece longitudinalmente el tallo y forma en el extremo una inflorescencia.

- Hojas: espinosas que miden 30-100 cm de largo.

- Flores: de color rosa y tres pétalos que crecen en las axilas de unas brácteas apuntadas, de ovario hipógino. Son numerosas y se agrupan en inflorescencias en espiga de unos $30 \mathrm{~cm}$ de longitud y de tallo engrosado.

- Fruto: las flores dan fruto sin necesidad de fecundación y del ovario hipógino se desarrollan unos frutos

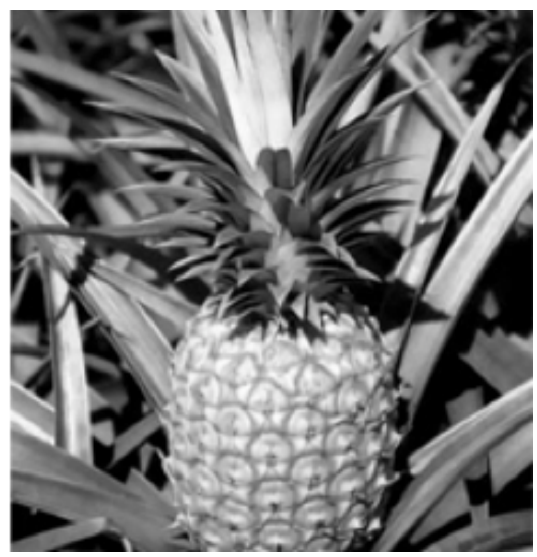

Figura 1. Piña Golden. en forma de baya, que conjuntamente con el eje de la inflorescencia y las brácteas, dan lugar a una infrutescencia carnosa (sincarpio). En la superficie de la infrutescencia se ven únicamente las cubiertas cuadradas y aplanadas de los frutos individuales.

\section{Clima y Suelo}

El cultivo de la piña se desarrolla en condiciones favorables, en altitudes que van desde 100 hasta 600 metros sobre el nivel del mar, aunque experiencias realizadas en diferentes áreas del país indican que se puede cultivar desde el nivel del mar.

Las temperaturas ideales a las cuales se desarrolla el cultivo oscilan entre $20^{\circ} \mathrm{C}$ y $30^{\circ} \mathrm{C}$, aunque temperaturas de $25^{\circ} \mathrm{C}$ a $27^{\circ} \mathrm{C}$ serían las óptimas para su crecimiento.

Precipitaciones entre 1.500 y 2.000 milímetros de lluvia anual son necesarias para garantizar un crecimiento normal del cultivo; en los periodos secos, utilizar riego complementario para no detener su desarrollo y en condiciones de exceso de lluvia, realizar prácticas de drenaje.

Los suelos con mejores condiciones para el desarrollo de este cultivo son los de textura liviana y bien drenados. La acidez $(\mathrm{pH})$ debe estar entre 4,5 y 6,0 con niveles muy bajos de elementos tóxicos como el aluminio.

Descripción del Cultivo de Piña Golden en el fundo Santa Teresa

Las actividades observadas en el fundo Santa Teresa comprenden desde la tala para incorporación de terrenos, pasando por la labranza primaria, secundaria selección de semillas, siembra y control fitosanitario durante el crecimiento, cubierta de la fruta para evitar la mosca de la fruta, cosecha y clasificación pos cosecha.

El fundo, con 203 hectáreas aptas para cultivo, posee solo 34 hectáreas del cultivo de piña siendo 28 hectáreas sembradas y 6 hectáreas de semilleros. Para producción de piña se necesitan climas tropicales. En la Tabla 1, se aprecia cómo son las temperaturas en la zona de Satipo.

\section{Labranza}

\section{Limpieza del Terreno}

Se realiza en dos modalidades: de un terreno nuevo a uno en cultivo o de uno ya habilitado y que ya cumplió su tiempo de descanso a fin de recobrar fertilidad. En el primer caso, se emplea el corte y quema (como en el caso de los árboles de naranjo) a fin de habilitar los campos para el cultivo de piña, empleando maquinaria pesada de movimiento de tierra, como el bulldozer, para arrancarlos de raíz y proceder a la uniformidad del terreno. Posteriormente, interviene la maquinaria agrícola. En el segundo caso, se aplica directamente maquinaría agrícola. Se comienza con pasada de rastras semipesadas de discos con los cuales se busca mullir e incorporar los restos de vegetales crecidos durante el descanso del terreno.

\section{Labranza primaria}

Generalmente comienza inmediatamente después de la limpieza del terreno con la pasada de un arado de discos a fin de lograr penetración y soltar terreno para la ejecución posterior de los camellones con una profundidad aproximada de $45 \mathrm{~cm}$. 
Tabla 1. Temperaturas del valle de Satipo

\begin{tabular}{|c|c|c|c|c|c|c|}
\hline \multicolumn{2}{|c|}{ Fecha Evaluada } & \multicolumn{3}{|c|}{ Temperatura ${ }^{\circ} \mathrm{C}$} & \multirow{2}{*}{$\begin{array}{c}\text { Humedad } \\
\text { Atmosférica } \\
\%\end{array}$} & \multirow{2}{*}{$\begin{array}{l}\text { Precipitación } \\
\text { mm/mes. }\end{array}$} \\
\hline Mes & Días & Máximo & Mínima & Media & & \\
\hline FEB 09 & 01 al 20 & 32,8 & 18,9 & 23,3 & 87,94 & 286,4 \\
\hline MAR 09 & 01 al 31 & 33,9 & 18,9 & 23,8 & 87,68 & 174,0 \\
\hline ABR 09 & 01 al 30 & 33,0 & 18,5 & 23,6 & 87,26 & 77,4 \\
\hline MAY 09 & 01 al 30 & 33,3 & 17,6 & 23,4 & 86,22 & 80,0 \\
\hline JUN 09 & 01 al 30 & 32,3 & 15,1 & 22,3 & 84,74 & 44,0 \\
\hline JUL $\quad 09$ & 01 al 31 & 32,6 & 15,6 & 22,6 & 84,25 & 70,6 \\
\hline AGO 09 & 01 al 31 & 34,2 & 17,1 & 24,0 & 79,54 & 19,2 \\
\hline SET 09 & 01 al 30 & 35,5 & 16,2 & 24,1 & 78,64 & 65,2 \\
\hline OCT 09 & 01 al 31 & 37,4 & 17,2 & 24,6 & 80,52 & 133,4 \\
\hline NOV 09 & 01 al 30 & 35,5 & 18,4 & 24,2 & 83,95 & 243,2 \\
\hline DIC 09 & 01 al 31 & 33,9 & 18,2 & 23,2 & 85,88 & 279,6 \\
\hline ENE 09 & 01 al 31 & 34,8 & 17,2 & 22,9 & 85,31 & 295,9 \\
\hline
\end{tabular}

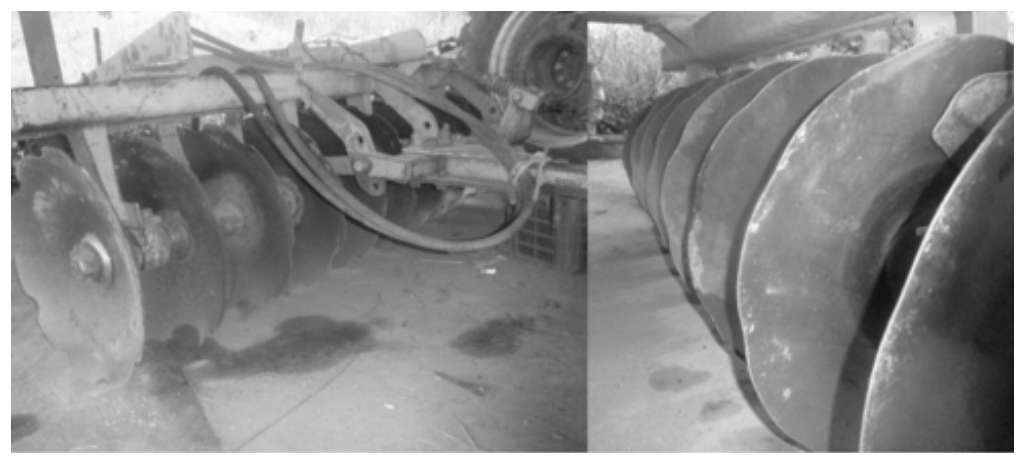

Figura 2. Rastras de discos semipesadas (FIANSA).

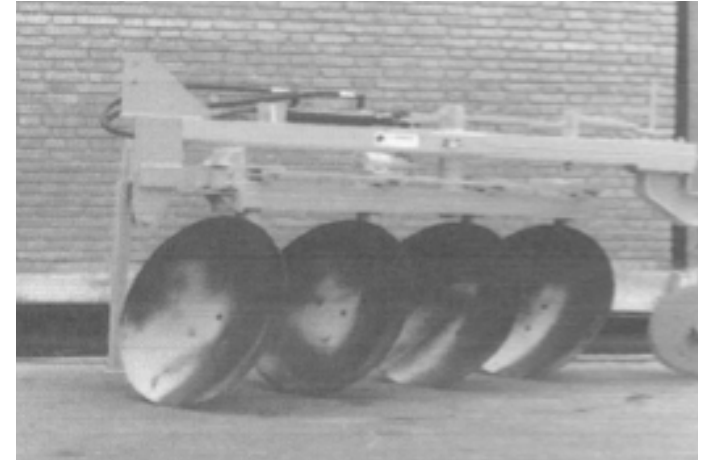

Figura 3. Arado de Discos (BALDAN).

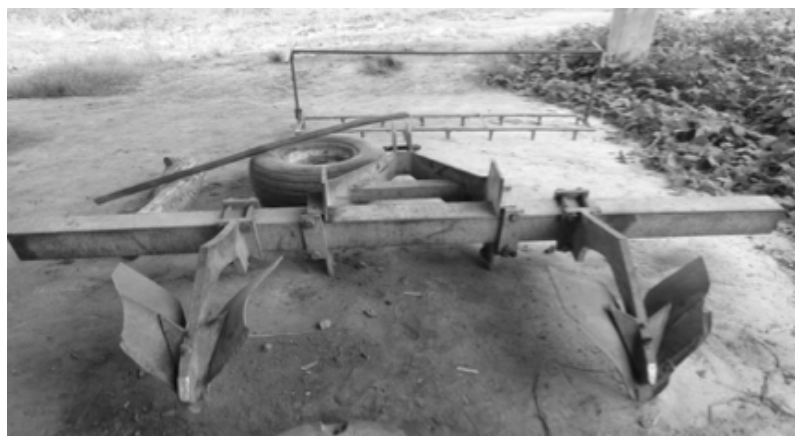

Figura 4. Surcador para cultivo de piñas.
El implemento con el que cuenta el fundo es un arado reversible de cuatro discos de 28 pulgadas de diámetro, marca Baldan hecho en Brasil. Con un ancho teórico de 1,10 metros. La potencia requerida es de 90HP aproximadamente.

\section{Labranza secundaria}

Después del barbecho o aradura, el terreno se encuentra con los prismas recién recortados y abrumados, debido al contenido arcilloso del terreno. Es necesario mullir el terreno a una profundidad aceptable que permita el amontonamiento de la cama en camellones para el depósito de la semilla. El gradeo o la acción para mullir el terreno se lleva a cabo utilizando la grada semipesada de 20 discos marca FIANSA, pasándola sobre el área en forma cruzada para un buen resultado. Por lo que el requerimiento es de aproximadamente 06 horas-tractor/hectárea.

El surcado es la actividad complementaria posterior al gradeo cuya finalidad es dejar preparada la cama para el depósito de los hijuelos de la piña, su profundidad es de aproximadamente $40 \mathrm{~cm}$. Se realiza con la ayuda de los cajones o surcadores preparados especialmente para este tipo de cultivo, en la localidad de Satipo (Hechizo) y que se muestra en la Fig. 4. Esta actividad requiere de 06 horastractor/hectárea, siendo la distancia entre surcos de 1,50 
metros.

\section{Siembra}

La modalidad de la siembra empleada es llamada en tres bolillas y consiste en depositar las semillas en dos hileras, en un mismo camellón a una distancia de:

Entre planta y planta à 0,30 metros.

Entre hileras à 0,40 metros.

Entre surco y surco à 1,5 metros.

Como se puede apreciar en la Fig. 5, esta modalidad de siembra permite colocar en una hectárea de cultivo 44000 plantas de piña Golden. Para sembrar la piña se utilizan semillas vegetativas (hija de la planta cosechada en la campaña anterior), la cuales se clasifican de acuerdo a su

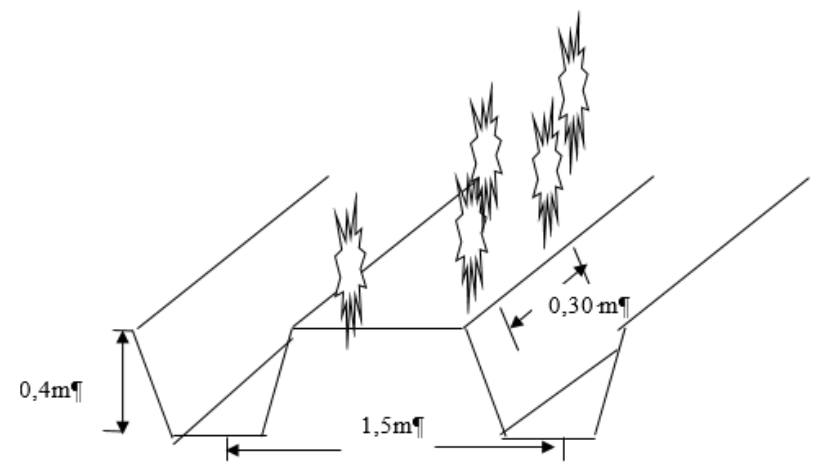

Figura 5. Diseño de siembra.

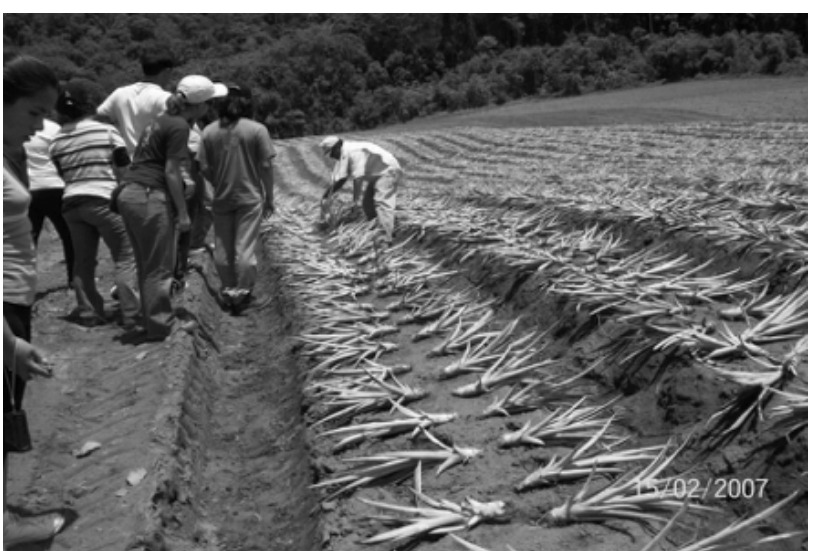

Figura 6. Distribución de semillas en el campo.

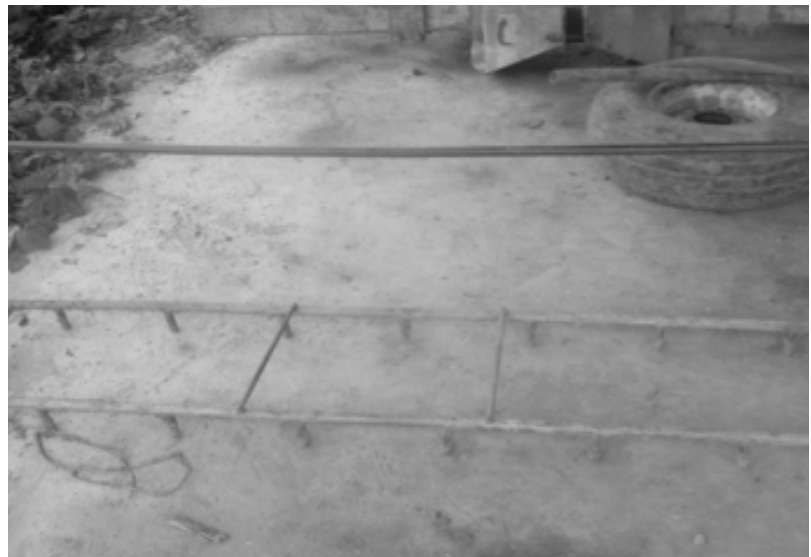

Figura 7. Plantilla de siembra. tamaño. Por cada planta se obtienen de 2 a 3 hijuelos; de ellos se extraerán unos 120 mil hijuelos para 3 hectáreas, aproximadamente, en la futura siembra. La altura de los camellones tiene que ver con la época, ya que en época lluviosa estos son más altos para que drene bien el agua.

La labor empieza con la marcación de los puntos de siembra, a fin de asegurar una distancia constante se emplea una plantilla construida e ideada en el fundo Santa Teresa, que colocada sobre el lomo del camellón permite marcar con una vara los espacios exactos donde se ubicarán los hijuelos. Seguidamente se distribuye las semillas como se puede apreciar en la Fig. 6.

Finalmente, una cuadrilla viene enterrando las semillas en el sitio exacto donde la plantilla las ubicó. La actividad de la siembra en el fundo Santa Teresa se realiza manualmente por lo que el requerimiento de trabajo es de 10 jornales/ hectárea ( 8 horas y media/día).

\section{Mantenimiento del cultivo y control fitosanitario}

Comprende las actividades durante el crecimiento y desarrollo de la piña Golden, entre ellas la de evitar la competencia de cualquier otra planta que no sea la de piña (malahierba), control que se hace mecánicamente mediante pasada de rastras cultivadoras o aplicación de herbicidas. El principal problema que tiene que afrontar el cultivo de piña es la presencia de la mosca de la fruta ya que debido al alto grado Brix que esta fruta presenta, asegura la presencia de este agente infeccioso. Aquí anotaremos algunos de los productos y tratamientos químicos que se emplean en esta etapa.

\section{Fertilización}

Los fertilizantes mayormente usados son: sulfato de calcio, de magnesio; cloruros, potasio, urea.

- A los 20 - 30 días de la siembra se le agrega fosfato de amónico (18\% N y $46 \%$ P) se aplica al pie de la planta en el lomo del surco, 10 gr por planta. No se puede aplicar en las costillas porque al momento del riego el agua se lo lleva.

- A los 2, 4 y 6 meses de trasplantado se le aplica urea y cloruro de amonio en una proporción de 1:1, 10 gr. por planta.

- A partir del $5^{\circ}$ mes se realiza la fertilización foliar, con una frecuencia de cada 15 días. Son 6 fertilizaciones antes de la inducción foliar. Luego de la inducción, son 3 fertilizaciones.

\section{Fertilizaciones foliares}

De seis a ocho meses antes de la inducción floral se aplica: urea, sulfato de potasio, nitrato de calcio, sulfato de zinc y Fertibagra (para que la planta no salga deforme) el cual aporta $10 \%$ de boro. El calcio debe ir siempre acompañado del boro para que genere un mayor movimiento de los azúcares.

Por cada hectárea del cultivo de piña, se siembra 44.000 plantas. Las cuales tendrán 2-4-6 meses de fertilización con urea-cloruro, a los 8-9 meses inducción floral lo cual da como resultado que la planta termine de florear a los 15 o 16 días. Como fertilizantes, en el fundo utilizan Fertibagra (10\% boro) para evitar la deformación del fruto, aplican urea en balde de $4 \mathrm{~kg}$ por cilindro (6 a 8 cilindros/ 
hectárea), Etetrel como producto para la inducción floral y la Dolomita para evitar la intoxicación de la planta por un exceso de $\mathrm{Al}$ en el suelo. En conclusión, por campaña utilizan cuatro fertilizantes en el suelo y nueve fertilizantes foliares, ya que la piña absorbe muchos nutrientes.

\section{La inducción foliar}

Para que floree uniformemente, se emplea Etrel (cuyo elemento activo es el etefón), etileno. Se mezcla $300 \mathrm{ml}$ por cilindro de 200 litros. O en tal caso también se disuelve urea, 1 balde de $4 \mathrm{~kg}$ por cilindro, y se aplica $15-20 \mathrm{ml}$. en la guía de la planta, 6-8 cilindros por ha. Esto se desarrolla manualmente.

Cuando en un muestreo se obtiene un $10 \%$ de insolación se realiza la aplicación, aproximadamente a los seis $u$ ocho meses del trasplante. Desde la inducción floral hasta la floración trascurren aproximadamente de seis a siete semanas.

\section{Floración}

La base del fruto empieza a florear primero.

A los ocho días empieza a florear la parte ecuatorial.

Por lo tanto entre los 15 y los 16 días termina de florear todo el fruto; cuando esto ocurre ya está formada la corona del grupo.

Durante este periodo generalmente atacan ciertas plagas y hongos, para lo cual también se realiza un control cada 8 días. El Teclabacilos es una plaga que ataca al fruto en el periodo de floración. Asimismo, se aplica glifosfatos en los caminos (herbicida).

\section{Brote del fruto}

Una vez terminada la floración comienza a brotar el fruto, que se cubre con una bolsa plástica de color blanco con pequeños agujeros que permitan respirar al fruto, a fin de reducir la infección con mosca de la fruta, se prefiere el color blanco ya que este color repele mejor a las mosca de la fruta (Melanoma viatix). Esta actividad es manual, las jornaleras embolsan 2.000 frutos aproximadamente en jornada de 8 horas y media. La maduración del fruto es a los 5 meses y medio.

\section{Tratamiento de hijuelos}

Estos se extraen de la axila de la planta, una vez recogido el fruto. En época lluviosa los hijuelos crecen más rápido

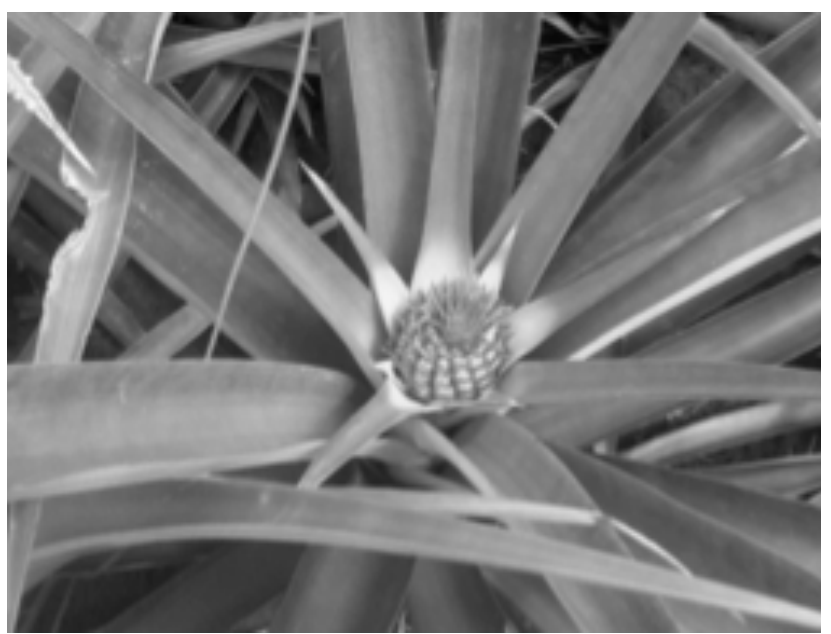

Figura 8. Floración de la Piña Golden.

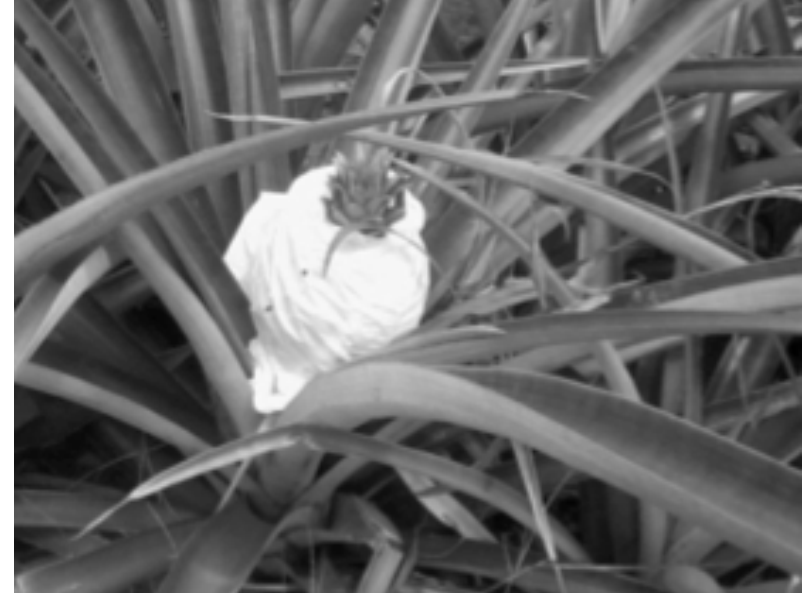

Figura 9. Embolsado del fruto contra la mosca de la fruta.

en comparación con las épocas secas. Se aplica vacuna para evitar las plagas, entre ellas la Cinamonis, hongo que produce la pudrición de la guía. Antes de trasplantar los hijuelos, se vacunan a los 30 y 60 días. La vacunación consiste en aplicar un fungicida, insecticida y fosfato de potasio que es un inductor de defensas en la planta. En el caso del fungicida se aplica un fosfitil aluminio $(1 / 2 \mathrm{~kg}$ por cilindro) y en el caso del insecticida se aplica dimetoato ( $1 / 2$ litro por cilindros de 200 litros.). Todo esto se aplica a cada planta en una cantidad de $15-20 \mathrm{ml}$ en la guía. En épocas lluviosas se realiza 3 aplicaciones ya que hay mayor probabilidad de encontrar enfermedades y en épocas secas 2 aplicaciones. Cada jornalero puede aplicar 2.500 semillas de hijuelos/jornal.

\section{Control de nematodos}

En cuanto a los nematodos, estos se comen las raicillas de las plantas por lo que también esto se controla mediante el encalado. Los géneros que más atacan son el pertilencius y el espertilencius. La cantidad de nematicida es de $40 \mathrm{~kg} / \mathrm{ha}$ por lo que a cada planta se le aplica aproximadamente $1 \mathrm{gr}$. Las aplicaciones se realizan en las mañana y en las tardes, en que el suelo contiene mayor humedad, siendo el medio más propicio para los nematodos.

Para el control fitosanitario se utiliza un "Aguilón", que es un equipo pulverizador (asperjadora de gran volumen), de la marca COINSA, con una capacidad de tanque de 2.000 litros que ha sido adaptado para la pendiente del terreno.

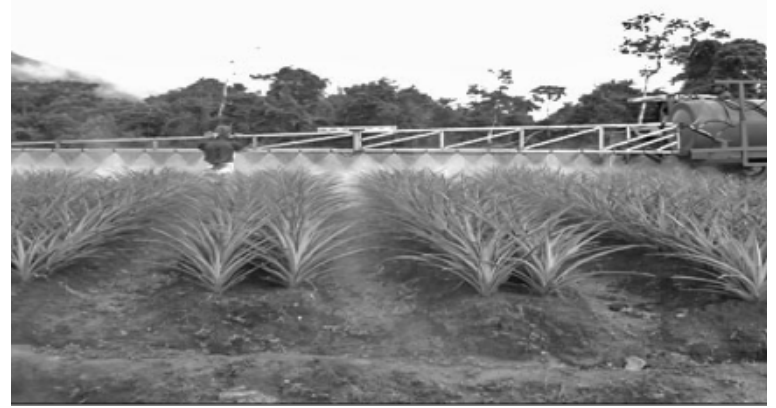

Figura 11. Asperjadora de gran volumen. 
Solo puede trabajar con un brazo de 13 metros de largo aproximadamente, ejerciendo las aplicaciones por ambos extremos logra abarcar un espacio de 25 metros con el respectivo traslape. Por lo que el terreno ha sido dividido por caminos de servicio para el tractor cada 25 metros con franjas de servicio de 5 metros aproximadamente. Debido a que el riego es por lluvia, se emplea un asperjador de cañón giratorio cuyo radio de acción es de 25 metros, que es utilizado en los meses de mayo y junio, o cuando se ausentan las lluvias.

\section{Principales productos químicos empleados}

Como cualquier cultivo, la piña necesita abono o sufre ataques de plagas, las cuales pueden causar un serio daño económico. Por este motivo mencionaremos a modo de recuento los principales productos químicos empleados en el cultivo de la piña Golden (nematicidas, insecticidas, fungicidas y herbicidas, etc.) a fin de mantener el cultivo en un buen estado fitosanitario.

Herbicida: Bibron.

Herbicida: Diuron.

Nematicida Sistémico: Mocap Etoprofos.

Fungi-Insecticida: Dimetoato (ingrediente activo es el Fosetal aluminio) este producto es usado para la desinfección de hijuelos.

Sulfato de potasio (abono).

Fosfato di amónico (abono).

Urea (abono).

Nitrato de calcio (abono).

Sulfato de calcio (abono).

Etrel (cuyo elemento activo es el etefón).

La floración de la piña dura entre 15 y 16 días, durante los cuales se aplica insecticidas fosforados, del órgano fosforado o del benzoato para la plaga del Thecla basilides llamada "Mariposa del fruto o Gusano barrenador de la piña". Se aplica con una frecuencia de 8 días.

Principales plagas de la piña golden en el fundo Santa Teresa.

Melasoma buculatrix (mosca de la fruta).

Helicotylenchus sp y Pratylenchus elegans (nematodos migratorios).

Triabioxis paradexus (hongo que ataca en post cosecha). Tecla basidia (barrenador de frutos).

Phytophtora cinnamomi (Pseudohongo).

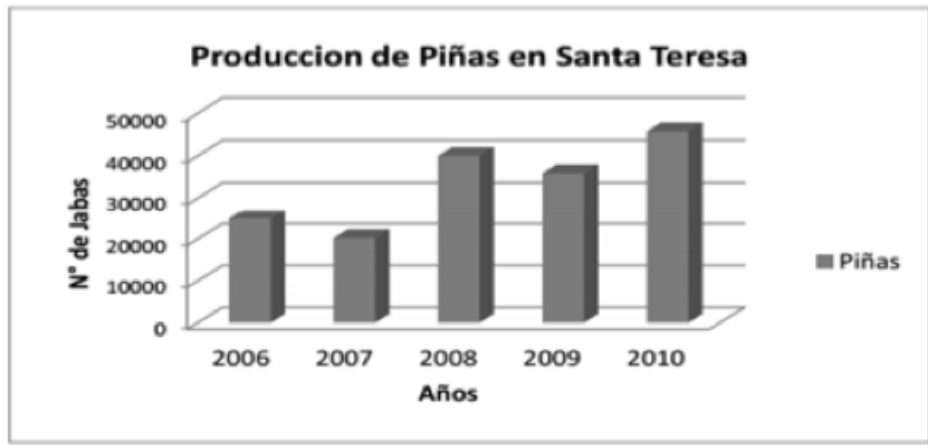

Figura 12. Producción de piñas.

Tabla 2. Producción de piñas en el fundo Santa Teresa

\begin{tabular}{lccccc}
\hline \multicolumn{1}{c}{ Mes } & $\mathbf{2 0 0 6}$ & $\mathbf{2 0 0 7}$ & $\mathbf{A n ̃ o}$ & $\mathbf{2 0 0 8}$ & $\mathbf{2 0 1 0}$ \\
\hline Enero & & 2,998 & 637 & 2,574 & 5,628 \\
Febrero & 2,295 & 2,720 & 480 & 3,128 \\
Marzo & 1,823 & 1,159 & 0 & 3,613 \\
Abril & & 1,124 & 3,150 & 224 & 2,756 \\
Mayo & 880 & 3,243 & 3,419 & 1,437 \\
Junio & & 2,687 & 7,617 & 2,054 & 3,408 \\
Julio & 1,835 & 4,425 & 1,859 & 2,846 \\
Agosto & 1,086 & 3,173 & 4,291 & 2,277 \\
Setiembre & 2,646 & 5,890 & 4,165 & 4,004 \\
Octubre & 2,989 & 1,235 & 3,531 & 2,219 & 7,688 \\
Noviembre & 8,007 & 664 & 1,168 & 4,314 & 7,212 \\
Diciembre & 2,949 & 1,419 & 3,247 & 10,283 & 1,750 \\
\hline Total & 6,316 & $\mathbf{2 0 , 2 0 5}$ & $\mathbf{3 9 , 9 6 0}$ & $\mathbf{3 5 , 8 8 2}$ & $\mathbf{4 5 , 7 4 7}$ \\
\hline
\end{tabular}

Nota: Unidad expresada en jabas de $18 \mathrm{~kg}$ c/u en promedio.

Fuente: Dirección del IRD-Selva Satipo, 2011. 


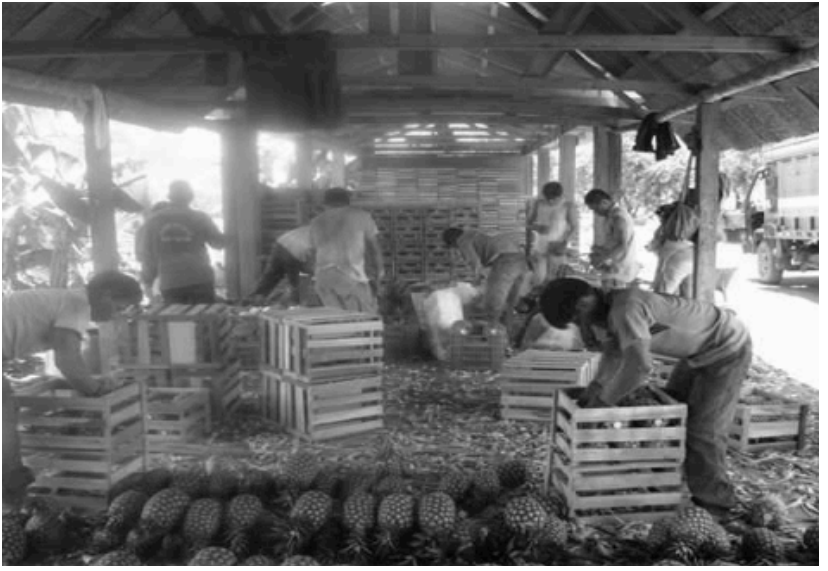

Figura 13. Selección y embalaje de piñas.

La cosecha es la actividad posterior a todas las ya realizadas previamente, comprende la recolección, acopio y clasificación para su distribución. Las piñas, al ser envueltas en bolsas plásticas, tienen que pasar aproximadamente unos 5 meses hasta que maduren. Estas no lo hacen homogéneamente, lo que dificulta el uso de maquinaria porque se cosecharían productos maduros y otros verdes lo cual traería pérdidas económicas.

Por otro lado, la cosecha manual o artesanal es un proceso tedioso, cansado y con gran pérdida de tiempo, ya que el trabajador tiene que ir fijándose piña por piña en el tamaño y color del fruto para verificar cuales están maduros para poder cosecharlos.

\section{Resultados y discusión}

En la labranza primaria, la acción de aradura requiere de 06 a 10 horas-tractor/hectárea. Esto debido a que se hará siguiendo las sinuosidades del terreno. En la labranza segundaria, la actividad requiere de 06 horas-tractor/ hectárea, siendo la distancia entre surcos de 1,50 metros.

La actividad de la siembra en el fundo Santa Teresa, se realiza manualmente por lo que el requerimiento de trabajo es de 10 jornales/hectárea ( 8 horas y media/día). En la fertilización cada jornalera fertiliza 1.000 plantas (10 gr por cada planta), 1 jornal de 3 a 4 horas.

La cosecha depende de la cantidad de plantas por hectárea (densidad de siembra). La densidad de siembra manejada es de 44.000 plantas/hectárea, como cada planta permite cosechar solo un fruto, entonces se obtienen 44.000 piñas como máximo. Si cada piña pesa entre un kilo y 1,5 kilos, entonces la cosecha será de aproximadamente 60 toneladas. Mensualmente se preparan dos hectáreas, a fin de que cada semana se coseche piñas. Todo tiene que estar programado para asegurar el abastecimiento del mercado durante todo el año. La recolección de los frutos se realiza con cuadrillas de trabajadores, que emplean jabas plásticas donde depositan los frutos maduros para trasportarlas hasta la carreta enganchada a un tractor (New Holland) y de allí al galpón de embarque, donde se selecciona y se clasifica de acuerdo a su tamaño y peso en jabas o cajas de maderas, de 8,10 o 12 piñas; también se evalúa el estado de la piña para su traslado a Lima. La venta se hace por calibre:

8 piñas por c/madera....S/. 24,00.à en época de enerofebrero está entre $\mathrm{S} / .35 \mathrm{y} \mathrm{S} / .40 .00$

10 piñas por $\mathrm{c} /$ madera...S/. 20.00

12 piñas por $\mathrm{c} /$ madera...S $/ .16 .00$

Por cada 1.000 plantas el peso del producto disminuye en 40 gr.

Costos.

Mencionaremos aquellos costos en donde los costos operativos, así como los costos fijos ya están involucrados, de tal manera que el lector con un poco de paciencia, podrá calcular el costo de producción de la piña Golden en el fundo Santa Teresa.

Para la realización de estas actividades se tienen los siguientes gastos:

Tractor (por hora)........... S/. 80.00,00

Combustible por galón....... S/. 14.50,00

Jornal de 8 horas diarias..... S/. 30.00,00

Millar de bolsas blancas..... S/. 30.00,00

El costo de embolsado es de S/. 3.000,00 soles por hectárea.

\section{Costo de Producción}

El costo de producción es de aproximadamente S/. $32.000,00 /$ hectárea, si se compra los hijuelos a S/. 0,30 céntimos cada uno, haciendo un total de $\mathrm{S} / .12 .000,00$. Pero en el caso de que se tenga un campo semillero, producto de la primera cosecha, el costo de producción sería de unos $\mathrm{S} /$. 20.000,00 al evitar el gasto por la compra de hijuelos. De estos cálculos, deducimos que el ingreso bruto por ha es de S/.10.000,00 aproximadamente, lo cual hace del cultivo de la piña un negocio rentable, como lo muestra el incremento de su exportación en la Tabla 4.

Conclusiones

Las diferentes actividades agrícolas que intervienen en la producción de la piña Golden en el fundo Santa Teresa son: Limpieza de terreno, Labranza primaria (con arado de discos), labranza secundaria (mullido del terreno con grada de discos), siembra manual previa selección y tratamiento de la semilla, control de malezas, control fitosanitario (con asperjadora de gran volumen adaptado aquí en el fundo), fertilización, riego por lluvias (ayudado por cañón asperjador), cosecha manual (acopio de los frutos maduros

Tabla 3. Producción de piña en las provincias de Chanchamayo y Satipo

\begin{tabular}{lccccc}
\hline Provincias & No de Productores & Ha en Verde & Ha en Producción & Rdto/ha & $\begin{array}{c}\text { Volumen de } \\
\text { Producción } \\
\text { TM }\end{array}$ \\
\hline Satipo & 1180 & 2360 & 1467 & 20,86 & 19700,8 \\
Chanchamayo & 3895 & 5843 & 5026 & 13,17 & 63207,36 \\
\hline Total & $\mathbf{5 0 7 5}$ & $\mathbf{8 2 0 3}$ & $\mathbf{6 4 9 3}$ & $\mathbf{1 7 , 0 1}$ & $\mathbf{8 2 9 0 8 , 1 6}$ \\
\hline
\end{tabular}


Tabla 4. Exportación de piña

\begin{tabular}{|c|c|c|c|c|c|c|}
\hline \multirow[b]{2}{*}{ MES } & \multicolumn{3}{|c|}{2011} & \multicolumn{3}{|c|}{2010} \\
\hline & FOB & Kilos & $\begin{array}{l}\text { Prec. } \\
\text { Prom }\end{array}$ & FOB & Kilos & $\begin{array}{l}\text { Prec. } \\
\text { Prom }\end{array}$ \\
\hline Enero & 14,564 & 15,839 & 0.92 & & & \\
\hline Febrero & 9,090 & 10,878 & 0.84 & 1,745 & 54 & 32.32 \\
\hline Marzo & 38,177 & 1,092 & 34.97 & 8,532 & 264 & 32.32 \\
\hline Abril & 18,086 & 495 & 36.51 & 2,678 & 1,566 & 1.71 \\
\hline Mayo & 23,758 & 667 & 35.61 & 144 & 7 & 20.03 \\
\hline \multicolumn{7}{|l|}{ Junio } \\
\hline Julio & & & & 3,491 & 108 & 32.32 \\
\hline Agosto & & & & 970 & 30 & 32.32 \\
\hline Setiembre & & & & 31,096 & 19,345 & 1.61 \\
\hline Octubre & & & & 395 & 95 & 4.14 \\
\hline Noviembre & & & & 27 & 12 & 2.19 \\
\hline Diciembre & & & & 24,391 & 31,701 & 0.77 \\
\hline TOTALES & 103,675 & 28,971 & 3.58 & 73,469 & 53,182 & 1.38 \\
\hline PROMEDIO MES & 20,735 & 5,794 & & 6,122 & 4,432 & \\
\hline \% CREC. PROM. ANUAL & $239 \%$ & $-5 \%$ & $-100 \%$ & $-52 \%$ & $-66 \%$ & $-100 \%$ \\
\hline ACUMULADO A MAYO & 103,675 & 28,971 & 3.58 & 13,100 & 1,891 & 6.93 \\
\hline \% CREC. ACUM. MAYO & $691 \%$ & $1432 \%$ & $-48 \%$ & $-84 \%$ & $-98 \%$ & $794 \%$ \\
\hline
\end{tabular}

empleando una carreta tirada por el tractor), posterior selección y clasificación de los frutos hasta el embarque hacia los mercados de Lima.

Se mencionan los principales productos de abonamiento, fertilización y control de plagas empleados en el fundo Santa Teresa, así como las dosis empleadas. Siendo la recomendación, para una buena instrucción del uso de estos productos químicos, recurrir directamente a la sapiencia de los técnicos del fundo. Es importante resaltar la necesidad de abonamiento con los productos NPK (Nitrógeno, Potasio y Fósforo), siendo los requerimientos del Nitrógeno y el potasio en los primeros estadios del cultivo y el de los productos fosforados para el fortalecimiento del fruto.

La revisión de información existente en otras partes del mundo donde se cultiva la piña Golden, y la información compilada in situ por las visitas de estudio del curso de Mecanización Agrícola (2008-2012), brindan un panorama de un futuro promisorio para el cultivo de piña Golden; esto aunado a factores de clima y mercado presentes en el valle de Satipo se convierten en una ventaja y esperanza alternativa al cultivo de la coca.

\section{Agradecimiento}

Al cariño y esfuerzo de los alumnos del curso de mecanización agrícola de la Universidad Nacional Agraria La Molina, que colaboraron con los datos para plasmar esta obra.

\section{Literatura citada}

Organismo Internacional Regional de Sanidad Agropecuaria [OIRSA]. (sf). Biblioteca Virtual. Disponible en: http://www.oirsa.org/aplicaciones/ subidoarchivos/BibliotecaVirtual/MANUALPINA.pdf

Curso de Mecanización Agrícola. 2008-2012. Informes. 